\title{
Características Socioculturales de la Actividad Física en Tres Regiones de Colombia
}

Rocío Robledo-Martínez

Odontóloga. M. Sc. Salud Pública. Instituto de Salud Pública, Universidad Nacional de Colombia. E-mail: rociorobledom@yahoo.com.

Recibido 10 Mayo 2006/Enviado para Modificación 10 Junio 2006/Aceptado 30 Junio 2006

\section{RESUMEN}

Objetivo Se indagaron los aspectos socioculturales relacionados con la vida en comunidad y la actividad física en las regiones de Bogotá, Antioquia y Quindío.

Métodos Se realizó un estudio exploratorio, cualitativo, cuyo interés sobre el objeto de estudio se enmarcó en las relaciones culturales de las regiones indagadas.

Las estrategias de investigación utilizadas fueron observación en terreno, entrevistas individuales y participativas semi-estucturadas (fuentes primarias) y revisión documental relacionada con los procesos culturales de las regiones de estudio (fuentes secundarias).

Resultados Los resultados obtenidos fueron de dos tipos: comunes para las tres regiones y específicos para cada una de ellas teniendo en cuenta los procesos culturales y las dinámicas comunitarias propias.

Conclusiones Los elementos identificados mediante la observación en terreno y los hallazgos interpretativos resultantes del estudio de las entrevistas, fueron concordantes con la teoría y las experiencias previas de otros países, aportando adicionalmente nuevas consideraciones asociadas al contexto cultural y comunitario específico de las regiones indagadas.

Palabras Clave: Actividad física, cultura, Colombia (fuente: DeCS, BIREME).

ABSTRACT

Socio-cultural characteristics of physical activity in three regions of Colombia 
Objective Investigating socio-cultural aspects related to living in a community and physical activity in Bogotá and the departments of Antioquia and Quindío.

Methods An exploratory, qualitative study was carried out within the framework of the cultural relationships found in the regions being investigated.

The research strategies used were field-observation, semi-stuctured individual and participative interviews (primary sources) and documentary review related to the cultural processes employed in the regions being studied (secondary source).

Results Two types of results were obtained which were common for the three regions and specific for each of them, bearing in mind their own cultural processes and community dynamics.

Conclusions The elements identified by field-observation and interpretative findings resulting from studying the interviews agreed with the theory and prior experience of other countries, providing new considerations associated with specific cultural and community context of the regions being investigated.

Key Words: Motor activity, culture, Colombia (source: MeSH, NLM).

L a fuerza que desempeña la cultura en los estilos de vida de los individuos, es un tema de investigación que inquieta tanto al mundo científico como a todos los actores interesados en diseñar estrategias de promoción de la salud.

Las aproximaciones teóricas a la cultura son diversas e igualmente incontables los intentos de definirla, la cultura se refiere a conductas actitudes y pensamientos aprendidos en la sociedad, ya sea a través de tradiciones o a partir de innovaciones contemporáneas (1). Es un legado social inscrito en el marco de la historia y el territorio, es identidad cultural, sentido de pertenencia, religiosidades, simbologías, historias culturales regionales, rituales, consumos culturales, tradiciones político administrativas, lengua.

Al diseñar modelos de movilización social y promoción de la salud, se ha puesto en evidencia la importancia de tener en cuenta aspectos culturales con el fin de conferir validez a dichos modelos.

"Los modelos inscritos en una dimensión social presentan una orientación comprensiva de la salud como proceso histórico-social, y, por tanto, las estrategias de promoción son más amplias e integrales, en tanto interpretan estilos de vida y comportamientos en sus respectivos marcos sociopolíticos y culturales, involucrando múltiples autores, más allá del sector salud” (2). 
La práctica de la actividad física en forma regular es de gran relevancia para la salud de las personas, pues disminuye el riesgo de padecer enfermedades crónicas no trasmisibles; pero, si queremos disminuir las tasas de sedentarismo al igual que otras problemáticas comportamentales, es preciso estudiar abordajes de multinivel en dichas problemáticas. La estructura conceptual de la construcción de modelos con tales características y de este tipo de intervención multinivel considera que tanto factores individuales como ambientales se encuentran vinculados al comportamiento. La conducta es vista como afectada y afectando múltiples niveles de influencia.

Dichos niveles, según diferentes propuestas, incluyen como agentes de influencia del comportamiento, factores personales, interpersonales, institucionales y grupales, comunitarios y de políticas públicas. Sin embargo, es necesario advertir que un único modelo o teoría no tiene capacidad para entender la complejidad del cambio de la conducta (3).

La formación del pensamiento social debe entenderse como un proceso en el cual, nosotros individuos inmersos en una cultura y una sociedad damos cuenta de la realidad, de los acontecimientos y del mundo al cual pertenecemos. Interpretar como se ha forjado este pensamiento social nos aproxima al entendimiento de las conductas de los seres humanos y de sus realidades subjetivas (4).

El estudio del "pensamiento ingenuo", del "sentido común" en adelante se torna esencial. La identificación de la "visión del mundo" que los individuos o grupos llevan en si y utilizan para actuar o tomar posición, es reconocida como indispensable para entender la dinámica de las interacciones sociales y aclarar los determinantes de las prácticas sociales (5).

Las técnicas metodológicas de investigación cualitativa nos ofrecen herramientas valiosas para desarrollar aproximaciones específicas en las cuales el individuo es tenido en cuenta en el contexto de su vida cotidiana, donde lo que realmente interesa es el intercambio de sentidos y significaciones, de cómo la gente arma y crea significados sobre aspectos tales como el sedentarismo y la actividad física, teniendo en cuenta además las dinámicas comunitarias.

Indagar las representaciones sociales es una de las corrientes de la psicóloga social que está teniendo mayor aceptación en el mundo de la investigación, principalmente en Europa; este estudio se aproximó a esta corriente epistemológica aunque no de forma estricta, ya que llegar al reconocimiento de las representaciones sociales hubiese requerido un trabajo de campo de 
mayor permanencia; se tomaron algunos elementos de las mismas, buscando rescatar el sentido común, ese tipo de saber, producto de la experiencia y la cotidianidad, pues es este el que finalmente está primando.

\section{MÉTODOS}

Se desarrolló una investigación exploratoria de enfoque cualitativo sobre las relaciones culturales, comunitarias y de la actividad física, en las regiones Bogotá, Antioquia y Quindío, en Colombia; entendiéndose la región, para este estudio, como un espacio geográfico producto de la interacción de factores históricos, humanos, demográficos, divisiones político administrativas, que incluye costumbres, formas de pensar e historia de la vida cotidiana de sus gentes. Una región es una zona homogénea con unas características físicas y culturales distintas de las de las zonas vecinas (1).

Como método de acercamiento al objeto de estudio se utilizó la etnografía para examinar grupos locales de población en el contexto en el que viven y se utilizaron como estrategias de investigación la observación en terreno, entrevistas semi-estructuradas individuales y participativas y revisión documental relacionada con los procesos culturales de las regiones de estudio. Las reseñas resultantes del estudio de las entrevistas semi-estructuradas se trabajaron mediante el programa de análisis cualitativo Atlas ti.

Se hizo una selección aleatoria de ocho barrios por región, estrato tres, veinticuatro en total; los barrios escogidos se diferenciaron unos de otros en sus características urbanas relacionadas con la facilidad de tener o no tener acceso a espacios que promuevan la actividad física.

Los barrios seleccionados en cada región fueron los siguientes:

Bogotá D. C.:Prado Veraniego Norte, Timiza, Granada Norte, Villa del Río, Villa Elisa, Villa Mayor, El Tunal Oriental, Marco Fidel Suárez.

Antioquia: En el Municipio de Medellín: Campo Valdés, San Benito, Jesús Nazareno, Los Alcázares, Castilla. En el Municipio de Itaguí: Santa María \# 3, El Carmelo. En el Municipio de Envigado: El Dorado. En el Municipio de Bello: Niquía.

Quindío: En el Municipio de Armenia: Ciudad Dorada, Corbones, 7 de Agosto, Arrayanes, San Francisco, Mercedes del Norte. En el Municipio de Montenegro: La Soledad, Centro. En el Municipio de Quimbaya: Cincuentenario, La Paz etapas I y II. 
La tarea exploratoria del trabajo de campo mediante entrevistas, estuvo orientada a dar respuesta a dos grandes categorías de abordaje: Movimiento corporal y actividad física; y Características socioculturales y comunitarias, las cuales fueron construidas teniendo en cuenta los lineamientos epistemológicos indicados, el propósito y objetivos de la investigación y la teoría existente relacionada.

En la categoría movimiento corporal y actividad física se enmarcaron los siguientes conceptos:

- Actividad física, riesgo y estilos de vida.

- Prácticas saludables.

- Barreras y facilidades de contexto para la actividad física.

- Barreras y facilidades individuales y sociales para la actividad física.

- Concepto de actividad física, como es entendida la actividad física.

- Cuerpo: sentido de percepción de si mismos y del cuerpo.

En la categoría características socioculturales y comunitarias se enmarcaron los siguientes conceptos:

- Actividad física valores y normas sociales

- Habilidades de la comunidad.

- Liderazgo.

- Participación ciudadana

- Pertenencia sociocultural

- Identidad regional: Sentido de pertenencia con el lugar y las personas.

- Sentido de comunidad.

El ejercicio de exploración de fuentes secundarias, permitió establecer una relación entre los procesos culturales de las regiones y los elementos identificados mediante las estrategias de investigación utilizadas en el trabajo de campo.

\section{RESULTADOS}

Los resultados del trabajo de campo se presentan teniendo en cuenta la información obtenida mediante observación en terreno y el análisis de las entrevistas semi-estructuradas individuales y participativas.

Se realizaron 88 entrevistas, 30 participativas y 58 individuales para un total de 130 personas entrevistadas. Los entrevistados fueron hombres y 
mujeres cuya edad se encontraba en el rango de 25 a 50 años, aunque es necesario aclarar que en algunos casos se entrevistaron personas cuyas edades no correspondían a dicho rango, por tratarse de informantes clave, que podían brindar información valiosa con respecto a las categorías de estudio de la investigación; se entrevistaron presidentes de juntas de acción comunal, líderes comunitarios, líderes deportivos, amas de casa, tenderos, vecinos, trabajadores, otros.

Como producto del trabajo de observación en campo, se pudieron establecer las siguientes percepciones:

- La infraestructura propicia de los barrios relacionada con la actividad física facilita la práctica de la misma; en algunos barrios esta es favorable gracias a la intervención en el espacio público y a la renovación de parques y escenarios deportivos. Se consideró como estructura favorable de un barrio o municipio, la presencia de áreas físicas como parques, canchas, polideportivos, salones comunales y espacios públicos que favorezcan la práctica de la Actividad Física.

- La asistencia de los institutos deportivos, está asociada a la infraestructura favorable del barrio, principalmente cuando hay grandes parques y polideportivos.

- Los barrios con estructura desfavorable tienen también los mayores problemas sociales y de ingreso familiar, a esto se le suma la menor presencia institucional. Es decir se unen factores como la pobreza, los problemas sociales, las barreras de contexto físico para la actividad física, la inseguridad y otros, afectando severamente la posibilidad de practicar actividad física. A pesar de que todos los barrios visitados eran de estrato 3 , existen marcadas diferencias socioeconómicas y urbanísticas entre ellos.

- Las características del barrio tales como conjuntos residenciales cerrados, caños, cañadas, grandes avenidas, estructuras topografías irregulares y andenes estrechos que segmentan el barrio, dificultan la práctica de la actividad física además impide la creación de ciclorutas.

- El encerramiento de los parques y su respectiva vigilancia le brinda a los usuarios una sensación de seguridad que favorece la rutina de la actividad física.

- Los parques que no están cercados no pueden ofrecer un sistema de seguridad óptimo lo cual desfavorece la actividad física principalmente en los niños.

- La placa deportiva o la cancha del barrio se convierten en el centro de congregación e interacción tanto deportiva como social 
- La mayoría de los barrios estudiados tienen fácil acceso al transporte público, lo cual desfavorece la actividad física. Los barrios con sistemas de transporte cercanos o inmersos en los barrios desestimulan la práctica de caminar.

- Las ciclovías tienen gran aceptación en todos los sectores y se constituyen en una alternativa para la práctica.

- La presencia de instituciones deportivas garantizan diferentes programas de recreación y deporte.

- Los barrios con deficiente infraestructura que favorezca la actividad física, tienen poca presencia de Instituciones deportivas. En este tipo de barrios hay poca movilización para la actividad física.

- La presencia de excelentes características estructurales del barrio que favorecen la actividad física, hace emerger categorías tales como la "pereza” o desmotivación.

- Adecuar espacios para el parqueadero de bicicletas facilita y fomenta el uso de ciclorutas.

Como producto del análisis de las entrevistas semi-estructuradas individuales y participativas se pudieron establecer hallazgos interpretativos que se presentan a continuación enmarcados según las categorías de abordaje previstas antes de iniciar trabajo de campo.

Hallazgos interpretativos sobre movimiento corporal y actividad física, comunes a las tres regiones:

Actividad física, riesgo y estilos de vida: Las personas manifestaron conocer el riesgo de llevar una vida sedentaria, a pesar de no ser activos físicamente. Creen que el deporte y la actividad física contribuyen a la prevención de algunos "vicios" como el alcoholismo y la drogadicción principalmente entre los jóvenes y que esta debe ser fomentada desde la niñez; además la actividad física es vista como una alternativa de uso productivo del tiempo libre.

Muchos de los entrevistados mayores refirieron conocer los beneficios de la actividad física porque el médico se los ha dicho y relataron que iniciaron una vida físicamente activa por recomendaciones hechas durante la consulta; su interés en estos casos radica en la preocupación de "enfermar" o de "sufrir un infarto"; adicionalmente reconocieron los beneficios estéticos y psicológicos que les ha brindado el tener una rutina diaria de actividad física.

Los entrevistados mostraron manejar el significado del término sedentarismo; pero, por el contrario, no poseían claridad en cuanto al concepto de 
actividad física, confundiéndolo con prácticas como el deporte o el ejercicio programado. Las mujeres expresaron verse a si mismas como sedentarias, los hombres también manifestaron verlas como menos activas que ellos.

Barreras y facilidades de contexto para la actividad física: La violencia y la delincuencia, fueron referenciadas como una de las barreras más importantes para realizar actividad física.

La percepción de seguridad fue considerada como muy importante para que las personas sean activas físicamente. La inseguridad percibida es más fuerte en los barrios con infraestructura desfavorable, aunque también en barrios con estructura favorable, pues los habitantes de estos barrios dijeron tener conocimiento de atracos e incluso enfrentamientos de pandillas y muerte entre los jóvenes. Se quejaron también de la invasión de los espacios deportivos por jóvenes que consumen alcohol y drogas. Los habitantes de algunos barrios exteriorizaron que esta es una de las causas por las cuales muchas personas abandonan prácticas como caminar a ciertas horas por las calles o frecuentar ciertos lugares del barrio.

El cerramiento de los parques fue señalado como una alternativa para mejorar condiciones de seguridad y de mantenimiento ambiental pues no solo, según sus palabras los protege en su seguridad personal sino que encuentran particularmente útil esta medida ya que "las personas que le tienen miedo a los perros no van a sufrir sustos y que además los amos descuidados que no vigilan el comportamiento de sus mascotas, ni recogen sus excrementos, van a ser controlados".

Los sujetos declararon también como barrera para realizar actividad física la invasión comercial del espacio público y calificaron esta situación como una de las causas para que los habitantes de la comunidad no "tengan conciencia” de la necesidad de espacios para la recreación y el deporte, pues "solo están pensando en sus negocios".

Los participantes reseñaron que la falta de espacios públicos para la recreación y el deporte tanto en los barrios como en las escuelas no solo se convierte en una barrera para la práctica de la actividad física, sino también genera otros problemas de carácter social.

Barreras y facilidades individuales y sociales para la actividad física: En términos generales los individuos también manifestaron que otras de las razones más importantes para no tener actividad física son aquellas que están directamente relacionadas con las circunstancias inherentes de su diario vi- 
vir, como son, la falta de dinero (motivo por el cual les es necesario "trabajar duramente”, sin poder gozar de tiempo libre); pereza (falta de motivación), falta de apoyo familiar o grupal, "compañía". La invitación puerta a puerta por parte de vecinos y amigos favorece la práctica de la actividad física. También argumentaron problemas de salud.

La prioridad presentada por las personas en el estudio, fue el trabajo y su situación económica. Para esta categoría las barreras para la práctica de la actividad física que se mencionaron con mayor frecuencia fueron en su orden: tiempo, dinero, pereza (falta de motivación), falta de compañía (soporte social), inseguridad, discapacidad, otros.

La condición de ser mujer trabajadora fue considerada por las mismas mujeres como una barrera, pues muchas son madre cabeza de hogar y en su cotidianidad deben desempeñar múltiples roles (trabajo de donde deriva su sustento, labores domésticas, crianza de los hijos) puntualizando además que no tienen con quien dejar sus hijos. El soporte familiar, social o institucional son alternativas para superar dicha barrera que puede ir desde el acompañamiento, la distribución de tareas en el hogar, hasta el reemplazo del cuidado de los niños mientras se tiene actividad física y otros.

Las personas que declararon no tener tiempo libre para realizar actividad física no tenían presente otras alternativas de su vida cotidiana para ser activas.

Ante la pregunta de como empleaban los participantes el tiempo libre muchos de ellos respondieron que no tenían tiempo libre, que sus prioridades estaban enfocadas en conseguir el sustento diario En contraste muchos de ellos también respondieron que utilizan su tiempo libre viendo televisión, durmiendo, jugando videojuegos, visitando Internet o en el caso de la ciudad de Bogotá, jugando tejo y tomando cerveza; el poco tiempo libre que las personas decían tener, no es empleado en la actividad física, se puso en evidencia que no contemplan la actividad física como una prioridad en sus vidas.

Las personas activas físicamente aclararon que la práctica de la actividad física requiere de disciplina y autodeterminación, y que "cuando la gente dice que no tiene tiempo esto es una disculpa para justificar la pereza”.

En cuanto al ejemplo (aprendizaje por observación) los entrevistados expusieron que cuando uno de los padres o los dos son deportistas esto incentiva la práctica de actividad física en la familia. Igualmente cuando existe soporte familiar, cuando los padres llevan a sus hijos para que realicen acti- 
vidades deportivas, o cuando todos lo hacen en familia y que también es importante el ejemplo de hermanos mayores.

Las personas activas físicamente indicaron incluir en su cotidianidad un espacio para la actividad física. Las personas inactivas físicamente, no conocen los programas y manifestaron no conocer nada con respecto a la actividad física del vecindario.

Concepto de actividad física: El concepto actividad física es entendido de forma confusa. Se puso en evidencia que muchos asocian la actividad física con deporte o ejercicio programado. La mayoría de las personas no tienen presente las diferentes formas de realizar actividad física como caminar, desplazarse en bicicleta a sus lugares de trabajo, subir escaleras etc. Tampoco tienen claro cuanto tiempo de actividad física es necesario, para obtener beneficios. Algunos piensan que han estado inactivos por largos periodos o toda su vida y que ya no es posible ser o volver a ser activos. Otros piensan que la actividad física y el deporte son únicamente para las personas jóvenes.

El concepto actividad física apareció asociado al tipo de trabajo que se realiza y aunque las personas reconocieron que en las tareas cotidianas están teniendo actividad física, desconocen las características necesarias de esta actividad para obtener beneficios.

Cuerpo, sentido de percepción de si mismos y del cuerpo: Generalmente los hombres expresaron percibirse como "más deportistas" que las mujeres a quienes ellos califican de "perezosas," a su vez algunas mujeres declararon percibirse a si mismas de la misma manera. Las mujeres dijeron que los hombres se ven motivados para realizar actividad física, por competitividad y vanidad.

Las personas reconocieron la importancia de la actividad física para "mantener un cuerpo saludable", también hubo consenso en decir que la actividad física produce bienestar físico y mental y que ayuda a "relajarse y quitar el estrés y que el "deporte es salud".

Hallazgos interpretativos sobre características socioculturales y comunitarias, comunes a las tres regiones:

Actividad física valores y normas sociales: En las tres regiones los entrevistados declararon que disfrutar de la vida familiar es muy importante al igual que mantener vínculos familiares no solo con la familia nuclear sino con la familia extendida. 
El baile con fines recreativos, "rumbear", fue señalado como una práctica agradable el cual goza de una gran aceptación en la vida social de los colombianos.

El jugar microfutbol fue reportado como una práctica común principalmente ejecutada por hombres adultos y niños varones. Comunicaron que les es fácil cerrar una cuadra y "echarse un partidito”.

Habilidades de la comunidad: Los habitantes que informaron percibir sus barrios como unidos y solidarios para resolver problemas también revelaron que se sienten a gusto en estos, que luchan y se "mueven", por sus metas; en contraposición de aquellos quienes no se sienten a gusto con lo que su barrio les ofrece. Una de las habilidades comunitarias más generalizadas es el control social informal a través de las alarmas comunitarias.

Liderazgo: Las personas relataron atender con mayor confianza el llamado de la parroquia o el sacerdote de la comunidad. El sacerdote es considerado como un líder comunitario con alta capacidad de convocatoria, comunicación y credibilidad.

Participación ciudadana: La mayoría de los entrevistados opinaron que la actividad física crea lazos de interrelación personal y se convierte en un elemento facilitador de interacción social. Las mujeres activas físicamente manifestaron disfrutar mucho de la participación social que les brinda la actividad física grupal. Los espacios donde se llevan a cabo actividades deportivas y recreativas se convierten en espacios de interacción social.

Un buen número de entrevistados tanto hombres como mujeres mencionaron que prefieren realizar actividad física en compañía de otras personas como amigos, familiares o vecinos lo cual puede considerarse como un factor facilitador de soporte (familiar, social) para la práctica.

Pertenencia social e identidad regional: El sentido de pertenencia y de comunidad está asociado a barrios con mejores condiciones físicas y sociales.

Sentido de comunidad: En las regiones de Antioquia y Quindío se pudo constatar que existe un fuerte sentido de comunidad entre los vecinos de los barrios y más aún con los vecinos de la cuadra; situación que no se evidenció en Bogotá pues ellos declararon considerar sus comunidades como poco solidarias.

Los habitantes de los barrios exteriorizaron que entre más tiempo se haya vivido en un barrio o comunidad, mayor es el sentido de comunidad para la 
resolución de los problemas y movilización de recursos para cubrir necesidades. Se lamentan de que las personas nuevas no conocen el esfuerzo con el cual se han conseguido los diferentes servicios.

Soporte institucional: Los habitantes de los barrios asociaron la presencia de los institutos relacionados con el deporte con actividades que logran conseguir una interacción y movilización comunitaria, independientemente de la eficacia de las juntas de acción comunal.

Las personas dicen aprovechar las oportunidades que les brinda la empresa privada para realizar actividad física. En la ciudad de Bogotá, se mencionó el Éxito como uno de estos espacios. En el municipio de Itagüí la plaza Mayorista, "Orgullosamente Paisa" cumple esta función.

\section{DISCUSIÓN}

En este estudio se confirmó la importancia de ahondar en los aspectos culturales de las comunidades ya que estos se ven afectando o estimulando la práctica de la actividad física, tanto de regiones culturales globales, como particulares.

Los resultados obtenidos son concordantes con el bagaje teórico existente planteado por la División de Nutrición y Actividad Física del Departamento de Salud y Servicios Humanos del CDC, en cuanto a aspectos como percepción y expectativas de los individuos relacionados con la actividad física y asociados a la creación de ambientes que brinden soporte y favorezcan la práctica (6).

Se pudo identificar, que en cuanto a las barreras personales para realizar actividad física, las afirmaciones de nuestros entrevistados están acorde con los resultados de estudios hechos por el CDC, ubicándose en primer lugar la falta de tiempo, seguido de falta de recursos, influencia social y obligaciones familiares.

Igualmente se visibilizó la necesidad de crear ambientes físicamente favorables para promover la actividad física y estilos de vida saludable y también el establecimiento de soporte social institucional y familiar, aspecto que ha sido enfatizado sistemáticamente por muchos autores.

El soporte familiar para favorecer la práctica de la actividad física hizo parte de los discursos de los participantes del estudio, confirmando lo que la teoría y experiencias no exitosas han demostrado. Podemos sugerir entonces 
que las acciones sean dirigidas a la familia, teniendo a la madre como eje y multiplicadora de información, fomentando así las interrelaciones familiares para condicionar de estilos de vida saludables. Es importante posicionar a la familia como un canal de respuesta colectiva y fortalecedora de redes sociales para la resolución de problemas; como agente de salud y como unidad organizativa de la comunidad.

El concepto de cuerpo se ve influenciado por los aspectos culturales de las regiones indagadas. Las identidades culturales persisten, bajo formas modificadas y bajo nuevas configuraciones como por ejemplo la transculturización (7), imposición a través de los medios masivos de comunicación de imágenes que llegan hasta los hogares, introducen elementos ajenos invadiendo los modelos propios culturales. Los medios invaden los modelos culturales del consumismo.

El cuerpo no puede ser asumido distante de lo cognitivo, emocional, lúdico, ético y estético de los sujetos; por el contrario, leer cuerpo y ser en el mundo como construcción cultural, es comprender el sistema modelador que llena de sentido la realidad material del cuerpo (8).

En los discursos, el sentido de cuerpo aparece también asociado a la actividad lúdica y de recreación lo que también afirma Jodelet: "El concepto de cuerpo ha cambiado en las sociedades modernas. Ahora hay un mayor acercamiento a su corporalidad y se acepta más la dimensión del placer, por encima de los efectos negativos como el dolor” (9).

Con respecto a las características socioculturales y comunitarias de las regiones en estudio y teniendo en cuenta que para un nivel de intervención, la región y el territorio son vistos como lugares " tatuados por las huellas de la historia, de la cultura y el trabajo humano, objeto de representación y apego afectivo" (10), podemos destacar que aquellas comunidades con un mayor sentido de pertenencia e identidad cultural, ostentan una mayor capacidad de movilización, que se ve reflejada en el incremento de la participación ciudadana y la unión de esfuerzos interinstitucionales y comunitarios, que redundan en el mejoramiento del nivel y calidad de vida de dichas comunidades. Este es el caso de la región cultural de la gran Antioquia, donde el arraigado sentido de pertenencia los impulsa positivamente hacia la movilización social.

En este estudio se verificó la importancia del conocimiento previo de los procesos culturales de las regiones, el cual se desarrolló mediante la revisión de la literatura relacionada. Este ejercicio favoreció un mejor entendimiento 
de las comunidades en las cuales se realizó el estudio, pudiendo comprenderlas no solamente como un grupo de personas que comparten un espacio geográfico, sino también como una estructura social en la cual los individuos que la conforman, tienen en común, aspectos tales como la forma de entender su realidad cotidiana y sus modos de vida. Esta cognición nos permite diseñar interacciones con una mayor probabilidad de éxito; "Examinar los procesos culturales de las regiones nos ayudan a comprender las relaciones entre la cultura y las distintas dimensiones de estos procesos” (10).

Los Antioqueños se sienten parte de su región lo que los hace participar en alcanzar metas propuestas, manifestando sentimientos de solidaridad entre los habitantes de las comunidades visitadas. La participación de la población en el desarrollo de su región, incluye acciones de negociación con el Estado y desarrollo de acciones en salud, sin menoscabar la autonomía ni la manifestación cultural de dicha región.

En los discursos de los entrevistados, principalmente de aquellos que se encuentran vinculados con las instituciones encargadas de promover la actividad física, la recreación y el deporte, aparecen declaraciones que manifiestan la necesidad de capacitar líderes que se apropien de los procesos. Los autores afirman que la capacitación de líderes y agentes para la movilización y creación de proyectos como un proceso de aprendizaje construyen participación y ciudadanía. El fortalecimiento del liderazgo y organizaciones comunitarias desemboca en estrategias de acción.

Consolidar la identidad cultural en torno a características culturales comunes y desarrollar la conciencia que comparten los mismos problemas, puede lograr la solidaridad entre las personas, la unidad y el deseo de trabajar juntos para transformar la realidad.

Crear sentido de comunidad es acercar a los actores sociales relevantes en torno a los objetivos comunes y la visión de desarrollo de la comunidad, estimulándolos a compartir un proyecto de vida, disponiendo de sus recursos y capacidades para enfrentar de forma coherente los problemas de la comunidad.

Se pudo identificar en las instituciones encargadas de la recreación y el deporte, una tendencia a rescatar los juegos tradicionales como un vehículo para la recuperación de identidades, ya que "Las prácticas estético expresivas (música teatro o danza), recreativas y lúdicas en las comunidades barriales son modos de recobrar memorias o de tejer lazos de pertenencia con el territorio, esto es de rehacer identidades” (7). Es menester rescatar la 
identidad cultural en la comunidad, recuperar valores positivos de antaño, fortalecer el tejido social y construir un futuro creando espacios para la participación •

\section{REFERENCIAS}

1. Calle H, Morales J. Identidad Cultural e Integración del pueblo Colombiano, OEI, Bogotá; 1994.

2. Prieto A. Modelo de prevención de la salud, con énfasis en actividad física, para una comunidad estudiantil universitaria. Rev Salud Pública. Bogotá 2003; 5(3): 284-300.

3. Mc Leroy K. An ecological Perspective on Health Promotion Programs, University of North Carolina. Health Education Quarterly 1986; 15(4): 315-377.

4. Ibáñez T. Representaciones Sociales Teoría y Método. Ideologías de la vida cotidiana. Psicología de las representaciones sociales, Barcelona: Sendai; 1968.

5. Abric JC. Metodología de recolección de las representaciones sociales. Prácticas sociales y representaciones. México: Ed. Conyoncán, S.A. de C.V.; 1994.

6. U.S.Department of Health and Human Services. Physical Activity and Health: A Report of the Surgeon General. Atlanta: USDHSS/CDC; 1996.

7. Barbero J. Un Nuevo mapa cultural, pensar nuestra multiculturalidad. T.M. Editores, Bogotá. 1998.

8. Lora J. La educación Corporal. Barcelona: Paidotribo; 1990.

9. Jodelet D. La representación social: fenómenos concepto y teoría. En: Moscovici S. Psicología social II. Barcelona: Ediciones Paidós; 1986.

10. Gimenez G. Territorio, cultura e identidades. La región sociocultural. En: Barbero J, López de la Roche F, Robledo A (Eds). Cultura y Región. Bogotá: Universidad Nacional de Colombia, Facultad de Ciencias Humanas, Centro de Estudios Sociales; 2002. 